\title{
Contributions of Strain Relaxation and Interface Modes to Thermal Transport in Superlattices
}

\author{
Vahid Rashidi \\ Department of Mechanical Engineering, University of Michigan, Ann Arbor, MI, 48109 \\ Kevin P. Pipe* \\ Department of Mechanical Engineering, University of Michigan, Ann Arbor, MI, 48109 \\ Department of Electrical Engineering and Computer Science, University of Michigan, \\ Ann Arbor, MI, 48109
}

\section{Abstract}

Superlattice structures are widely used in electronic and optoelectronic devices, many of which depend heavily on thermal management for performance and reliability. It has been observed that silicon/germanium superlattices exhibit an enhancement in thermal conductivity at very short period lengths, which has been attributed to the contribution of coherent phonons. Here we investigate additional potential contributions to enhanced thermal conductivity in superlattices as period length is reduced, finding that a reduction in strain relaxation as well as increased contributions of interface modes that have vibrational character intermediate between those of the two constituent materials offer additional mechanisms for increased thermal conductivity.

\footnotetext{
*Corresponding author

Email addresses: vrashidi@umich.edu (Vahid Rashidi), pipe@umich.edu (Kevin P. Pipe )

URL: http://www.umich.edu/ vrashidi (Vahid Rashidi), http://www.umich.edu/〜 pipe (Kevin P. Pipe )
} 
Keywords: Superlattices, Heat transfer, Thermal boundary resistance, Kapitza resistance, Phonon, Molecular dynamics

\section{Introduction}

Superlattices have shown promise for applications such as thermoelectric devices [1], semiconductor lasers [2, 3], and transistors [4]. For these applications, thermal management is often important to performance and reliability; in the case of thermoelectric devices, heat transfer is key to fundamental operation. In general, thermal conductivity has been found to decrease in superlattices as period length decreases, due to increasing interface density [5]. Several studies however have indicated that thermal conductivity increases for very short period lengths, and have attributed this effect to phonon coherence $[6,8]$.

In addition to phonon coherence, other factors may play a role in increasing the thermal conductivity of short-period superlattices, such as a lack of strain relaxation that leads to a more uniform lattice constant. Lattice mismatch in epitaxial structures imposes a residual strain field that has a maximum at the interface and relaxes away from the interface [9]. It is well known that lattice strain can have a significant effect on the optical $[11,12,13]$ and electrical [10] properties of materials. Because the thermal properties of semiconductors are primarily dictated by phonons, the properties of which are affected by lattice strain, several studies have examined the effect of strain and stress on bulk and interfacial heat transfer $[14,15,16]$. Isotropic tensile strain of $\sim 3 \%$ was shown [17] to decrease the thermal conductivity of silicon by a factor of $\sim 0.82$. Experimental and numerical studies 
have also shown that compressive stress on a weak interface can increase its thermal conductance $[18,19]$. As the period length of a superlattice decreases below the strain relaxation length, the lattice constant within the superlattice is expected to fall within a narrower range, potentially leading to reduced scattering as phonons experience less variation in acoustic impedance. Furthermore, as the interface density increases, interfacial vibrational modes (which may have a character intermediate between those of the two constituent materials) are expected to play an increasing role in heat transfer and may create an additional channel for phonon transmission. To assess the contributions of these two factors to thermal transport in short-period superlattices, we apply an approach combining an initial energy minimization process (to accurately account for strain relaxation) followed by MD simulation (to analyze physical properties). This energy minimization method, which provides a means to generate strain profiles in an atomistic simulation that are in agreement with experimental results, can be used to study the physical properties (e.g., electrical, optical, and thermal) of strained interfaces; we use it here to study the effects of strain relaxation on thermal boundary resistance (TBR) and thermal conductivity in Si/Ge heterostructures and superlattices with various period lengths. We also calculate the phonon density of states (PDOS) for the materials in the superlattice to examine differences in PDOS that arise in superlattices with different period lengths, particularly the emergence of contributions due to interfacial (silicon-germanium) bonds in short-period superlattices. 


\section{Simulation Technique}

Several studies have examined the thermal conductance of a single heterointerface or the thermal conductivity of a multilayer heterostructure in materials systems that have significant lattice mismatch $[20,21,22]$. However, these and other previous computational studies have not accounted for strain relaxation when predicting the physical (e.g., optical, electrical, thermal) properties of a junction; instead, piecewise-constant strain have been specified before physical properties are calculated using techniques such as molecular dynamics (MD). This approach neglects the spatial variation of material properties such as phonon dispersion which may significantly affect the junction's properties. We implement a non-equilibrium molecular dynamics (NEMD) technique [23] with a Stillinger-Weber (SW) force field [24] to study thermal transport in $\mathrm{Si} / \mathrm{Ge}$ heterostructures. An initially unrelaxed piecewise constant strain profile similar to previous studies $[20,21]$ is first implemented in the simulation cells. Non-periodic boundary conditions are applied in all directions to mimic heat transfer in a nanorod and to let the strain relax in the system. For certain simulations a steepest descent (SD) energy minimization technique is applied prior to dynamic simulation in order to relax the strain in the system. The energy minimization stops when either the normalized change of the total potential energy of the system is less than $10^{-12}$ or the change in the total force vector is less than $10^{-12}(\mathrm{Kcal} / \mathrm{mol} \AA)$. Four layers of atoms on the periphery of the system are fixed in place to avoid the sublimation of atoms into vacuum during MD simulation. A time step of one femtosecond [6] is used to capture the fastest atomic vibrations in the system. A Langevin thermostat [26] is used to thermalize the simulation 


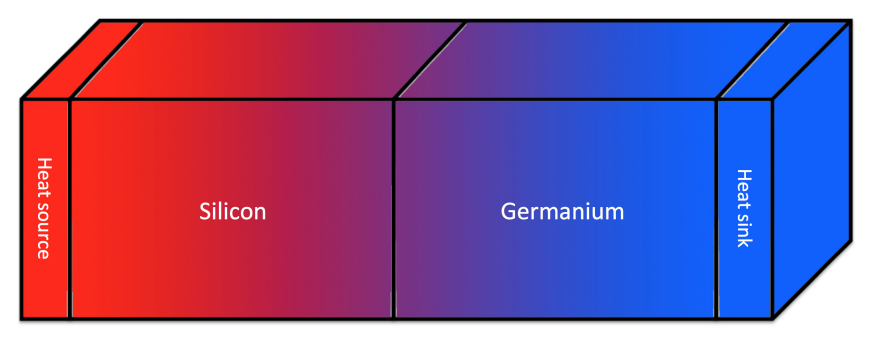

Figure 1: Single junction heterostructure simulation cell.

cell. The temperature of the system is first set to $5 \mathrm{~K}$ for $0.1 \mathrm{~ns}$ and then set to $50 \mathrm{~K}$ for $0.2 \mathrm{~ns}$ to relax the initial thermal noise in the system, after which the temperature is set to $500 \mathrm{~K}$ for $0.7 \mathrm{~ns}$. The thermostat is then removed and the system evolves in an NVE ensemble (constant number of particles, volume, and energy) in the presence of a constant heat flux that is applied through a heat source and heat sink to impose a temperature gradient. The system evolves in this ensemble for $6.5 \mathrm{~ns}$ to let the temperature gradient stabilize, after which the system evolves for one more nanosecond, during which the temperature gradient across the material is recorded to derive the thermal conductivity and/or thermal boundary resistance (TBR). Figure 1 schematically shows a single junction simulation cell. To achieve a good statistical average, the temperature profile of the system is averaged over the final one nanosecond (which corresponds to one million time steps). The TBR is calculated based on Fourier's law [23]:

$$
T B R=\frac{q}{\Delta T}
$$

where $\mathrm{q}$ is the heat flux passing through the interface, and $\Delta T$ is the temperature drop at the interface. To calculate the strain in the direction perpendicular to the interface, the position of the center of mass of each atomic 
layer is calculated at each time step, after which the change in distance between two adjacent layers with respect to the interlayer distance of the pure material at room temperature (one quarter of lattice constant of the pure material) is used to calculate the strain (Eq. 2).

$$
\varepsilon_{x x, a b}=\frac{\left(\frac{\sum\left(x_{a, i}\right)-\sum\left(x_{b, j}\right)}{(y * z * 2)}\right)}{S L}
$$

where $\varepsilon_{x x, a b}$ is the strain in the $x x$ direction (perpendicular to the interface) between adjacent layers $a$ and $b, y$ and $z$ are the number of cross sectional unit cells, $i$ and $j$ are the atom numbers in the $a$ and $b$ layers respectively, and $S L$ is the distance between the two layers in the unstrained pure materials at room temperature. For strain in the planes parallel to the interface, the average distance between the top and bottom atoms of each layer is used to calculate the strain. To verify the accuracy of our calculations, we first predict the lattice constants and thermal conductivities of pure silicon and germanium, using periodic boundary conditions to represent a bulk crystal. The Green-Kubo technique [23] is used to derive the thermal conductivities based on the averages of 21 calculations. The results are shown in Tables 1 and 2 and are in good agreement with literature values $[27,17,28]$. All MD simulations have been done using the LAMMPS (http://lammps.sandia.gov) package [29].

Table 1: Derived silicon and germanium lattice constants.

\begin{tabular}{cc}
\hline \hline Material & Lattice Constant $(\AA)$ \\
\hline Silicon & 5.433 \\
Germanium & 5.655 \\
\hline
\end{tabular}


Table 2: Derived silicon and germanium thermal conductivities.

\begin{tabular}{cc}
\hline \hline Material & Thermal Conductivity $(\mathrm{W} / \mathrm{mK})$ \\
\hline Silicon & $107 \pm 32$ \\
Germanium & $59 \pm 12$ \\
\hline
\end{tabular}

\section{Results}

Starting with a piecewise constant strain profile in the simulation cell and using our strain relaxation technique discussed above, we examine the relaxed strain profile in $\mathrm{Si} / \mathrm{Ge}$ heterostructures and the effect of this strain relaxation on thermal properties. The simulation cells used here consist of $10 \times 10 \times 288$ unit cells of atoms in the diamond cubic lattice structure $(8$ atoms in each unit cell).

\subsection{Strain Relaxation at Single Si/Ge Heterojunction}

For the same simulation cell we run two simulations: one with a conventional piecewise constant strain profile and one with the energy minimization step to relax the strain in the material prior to dynamical simulation. The initial piecewise constant strain profile is similar to those used previously $[20,21]$. Lattice mismatch between the materials causes strain in the direction parallel to the interface. Axial strain then arises as the system reduces its potential energy. The relaxed strain profile for a single heterojunction in the lateral direction is shown in Figure 2. The strain profile shows that most of the strain is relaxed in a few layers close to the interface $(\sim 2 \mathrm{~nm})$, while a small amount relaxes over a range of approximately $60 \mathrm{~nm}$. This latter relaxation is due to the fact that the global energy minimum is not 


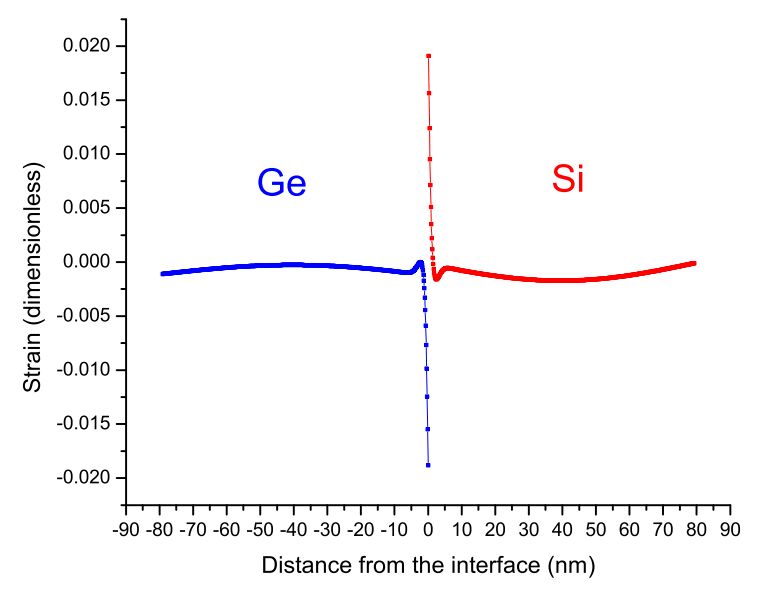

Figure 2: Relaxed lateral strain profile for a single heterojunction.

quite reached during energy minimization within a time that was practical for us to simulate ( $\sim 50 \mathrm{ps})$. Figure 3 illustrates the approach to the global energy minimum. We assume in what follows that the impact of this residual strain on heat transfer (and TBR in particular) is small compared to that of the large strain relaxation within a few nm near the junction.

Our post-relaxation strain profile at the junction is in good agreement with experimental results for strain magnitudes and relaxation lengths $[9,30]$.

\subsection{Strain Relaxation Effects on TBR}

Because of the sharp variations in material composition and strain that occur very close to the interface, a high scattering rate for phonons is expected. After thermalizing the system in an NVT ensemble (constant number of particles, volume, and temperature), an artificial temperature gradient is generated in the system by adding and removing heat using the reservoirs 


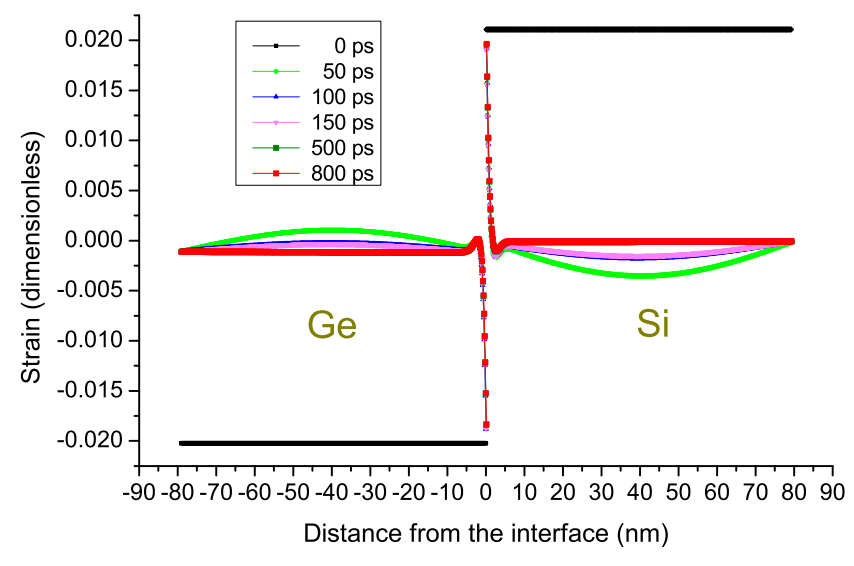

Figure 3: Lateral strain profile during strain relaxation. Legend indicates the time at which the strain profile is calculated during the relaxation process.

at the two ends. To calculate the TBR, best-fit lines for the temperature profiles in the two materials are calculated, and the discontinuity in these lines at the interface is used to derive the TBR using Equation 1. After simulating systems with piecewise constant strain profiles and systems with relaxed strain profiles, we find that the TBR values for these two strain conditions are not significantly different (Table 3), suggesting that TBR depends most on the lattice properties within a few nanometers of the interface and not particularly on the strain relaxation length. The fact that TBR values for systems with relaxed strain profiles are slightly higher than TBR values for systems with piecewise constant strain profiles suggests additional phonon scattering within the relaxation length.

Although literature values for TBR at nanowire heterjunctions are not available, both the order of magnitude of our TBR values and their trend 
Table 3: TBR values for different strain and temperature conditions.

\begin{aligned} \hline \hline Strain and Temperature Conditions & TBR $\left(\mathrm{W} / \mathrm{m}^{2} \mathrm{~K}\right) \\$\hline Piecewise constant strain $(600 \mathrm{~K}) & (2.85 \pm 0.02) \mathrm{e}-9 \\$ Relaxed strain $(600 \mathrm{~K}) & (2.96 \pm 0.13) \mathrm{e}-9 \\$ Piecewise constant strain $(500 \mathrm{~K}) & (2.91 \pm 0.09) \mathrm{e}-9 \\$ Relaxed strain $(500 \mathrm{~K}) & (3.07 \pm 0.17) \mathrm{e}-9 \\$ Piecewise constant strain $(300 \mathrm{~K}) & (3.62 \pm 0.05) \mathrm{e}-9 \\$ Relaxed strain $(300 \mathrm{~K}) & (3.77 \pm 0.09) \mathrm{e}-9\end{aligned}$

versus temperature are in good agreement with literature calculations of the TBR between two sheets of silicon and germanium [20].

\subsection{Strain Effects on Thermal Transport in Superlattices}

We have found above that strain at a $\mathrm{Si} / \mathrm{Ge}$ nanorod heterojunction relaxes away from the interface within a few nanometers. While it was also shown that this relaxation does not have a large impact on the TBR at the junction, it is also interesting to consider whether strain relaxation has an impact on the thermal conductivity of a superlattice in which the layer thicknesses are on the same order as the strain relaxation length. Such superlattices are often used in electronic devices such as thermoelectric devices [1]. To investigate this, we simulate thermal transport in nanorod superlattices of various period lengths having a total length of $\sim 160 \mathrm{~nm}$ and cross-sectional area of $4.43 \mathrm{~nm} \times 4.43 \mathrm{~nm}$. Using the energy minimization process described above, we arrive at the relaxed strain profiles shown in Figure 4. Figure 5 shows strain profiles both before and after relaxation in eight periods of a 


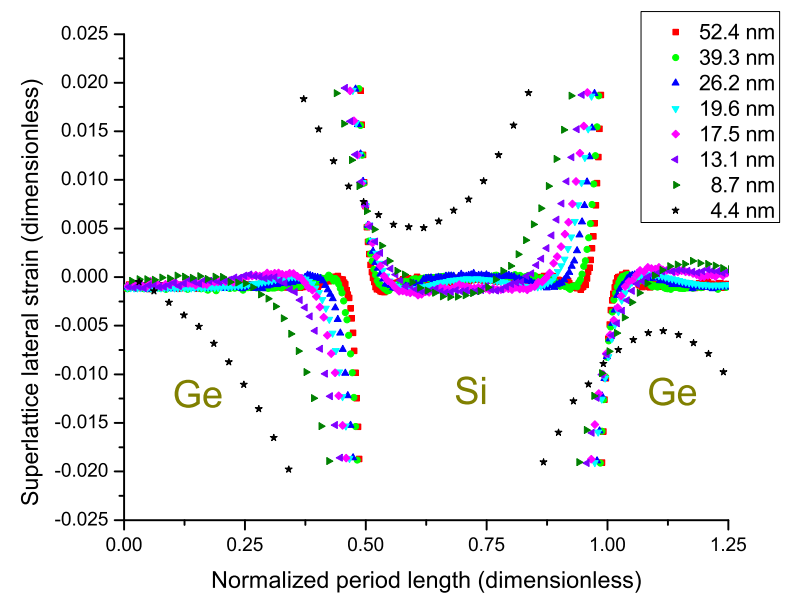

Figure 4: Lateral strain profiles in superlattices with different period lengths (indicated in the legend). For very short period lengths, the strain cannot fully relax, causing the structure to have a more uniform lattice constant. For illustration purposes, the period lengths are normalized by the length of each period.

superlattice having a period length of $20 \mathrm{~nm}$. Figure 6 shows the average lateral lattice constant in a representative region. From the figure it is clear that the average lattice constant is nearly uniform for very short period lengths due to a lack of strain relaxation. This uniformity suggests that less phonon scattering may occur, contributing to larger thermal conductivity.

We have calculated the thermal conductivities of superlattices with either relaxed strain profiles or piecewise constant strain profiles. The thermal conductivities of long-period superlattices are expected to be relatively insensitive to strain relaxation, since this relaxation happens over a very short length scale and TBR was shown above to be only slightly affected by nonzero strain relaxation length. The thermal conductivities of short-period 


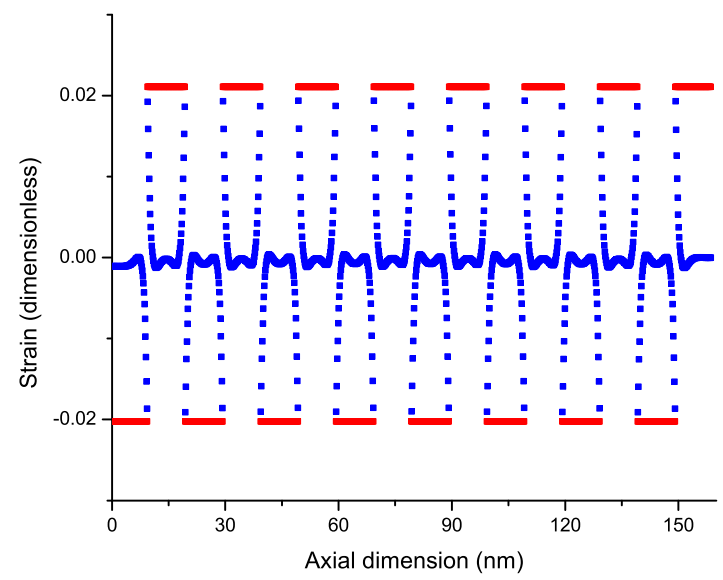

Figure 5: Lateral strain profiles before and after relaxation in eight periods of a superlattice having a period length of $20 \mathrm{~nm}$. The red lines show the initial strain profile and the blue curves show the post-relaxation strain profile.

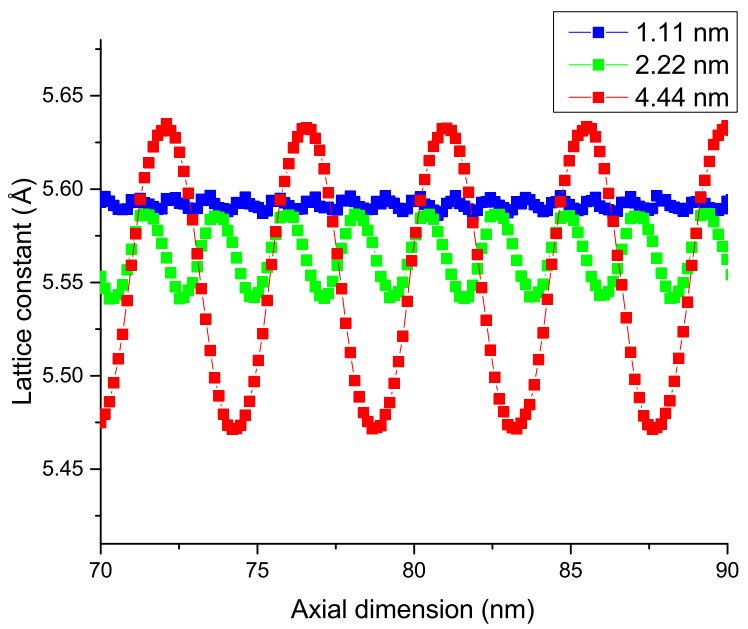

Figure 6: Average lateral lattice constant profiles after relaxation, showing uniformity in lattice constant for the smallest period lengths due to a lack of strain relaxation. 
superlattices are likewise expected to be similar for piecewise constant and relaxed strain profiles, as layer thicknesses less than the relaxation length do not allow the strain to fully relax. These expectations are confirmed in Figure 7. The superlattice exhibits a minimum in the thermal conductivity at a particular period length; a similar minimum has previously been observed $[6,8]$ and attributed to phonon coherence [6]. Interestingly, the period length associated with this minimum is very close to the strain relaxation length calculated above, indicating that the strain is not fully relaxed anywhere within the structure for period lengths smaller than this value. This leads to a more uniform lattice constant throughout, and consequently a presumable reduction in phonon scattering, which could contribute to the increase in thermal conductivity observed at small period lengths. To derive the TBR per interface in the superlattices, we first calculate the thermal conductivities of silicon and germanium nanowires with a length of 159.7 nanometers (the rest of the geometrical parameters were similar to those of the superlattices simulated). Calculated thermal conductivities of silicon and germanium nanowires were $24.75 \mathrm{~W} / \mathrm{mK}$ and $16.49 \mathrm{~W} / \mathrm{mK}$, respectively. Based on these values, we derived the average TBR per interface in various superlattices; the results are shown in Figure 8. We find that the TBR decreases with decreasing period length. It has previously been shown that the thermal conductivities of silicon nanowires are length dependent [7], with longer nanowires having a higher thermal conductivity. This suggests that the curvature in Figure 8 would have been even sharper had we instead used length-dependent thermal conductivities (which are difficult to calculate in NEMD for short nanowires) for silicon and germanium instead of constant 
values. The rapid reduction in TBR as the period length gets smaller in comparison to the less rapid rate of increase in the number of interfaces supports the enhancement of thermal conductivity for very short period length superlattices. The TBR values for short period lengths is more accurate because of less statistical error due to averaging over a larger number of interfaces; statistical error is expected to account for the large discrepancy in the system with the longest period length. We also calculated the percentage density of interface bonds (bonds between silicon and germanium) in superlattices with different period lengths, as shown in Figure 9. We see that the density of interface bonds increases rapidly at short period lengths, suggesting that the vibrational characteristics of these bonds may have an important bearing on the thermal conductivities of short-period superlattices, as discussed in the next section.

Through a Fourier transform of the velocity autocorrelation function, we calculate the phonon density of states (PDOS) for silicon and germanium in the superlattice structures for different period lengths in order to further understand the changes in superlattice thermal conductivity. A similar technique has previously been used $[31,32]$ to calculate PDOS for nanowires. Figure 10 shows the PDOS for silicon and germanium in superlattices with different period lengths.

We see the appearance of a peak between $12 \mathrm{THz}$ and $15 \mathrm{THz}$ in both silicon and germanium for superlattices with small period lengths. The introduction of this peak is due to an increased density of interface bonds, which contribute additional vibrational modes. As the number of interfaces increases, the density of interface bonds increases and hence the vibrational 


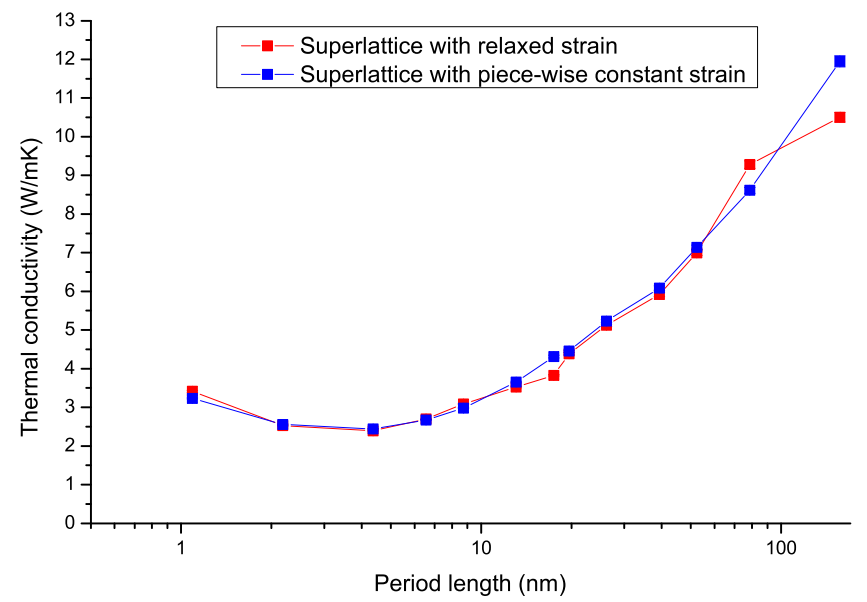

Figure 7: Superlattice thermal conductivity as a function of period length. The thermal conductivity exhibits a minimum at a period length approximately equal to twice the strain relaxation length.

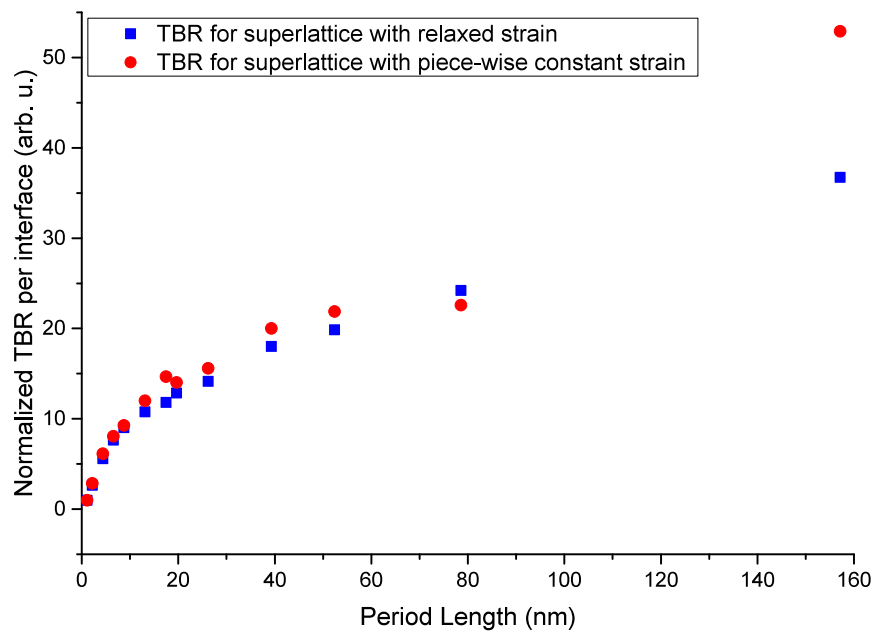

Figure 8: Calculated thermal boundary resistance per interface for superlattices with different period lengths. 


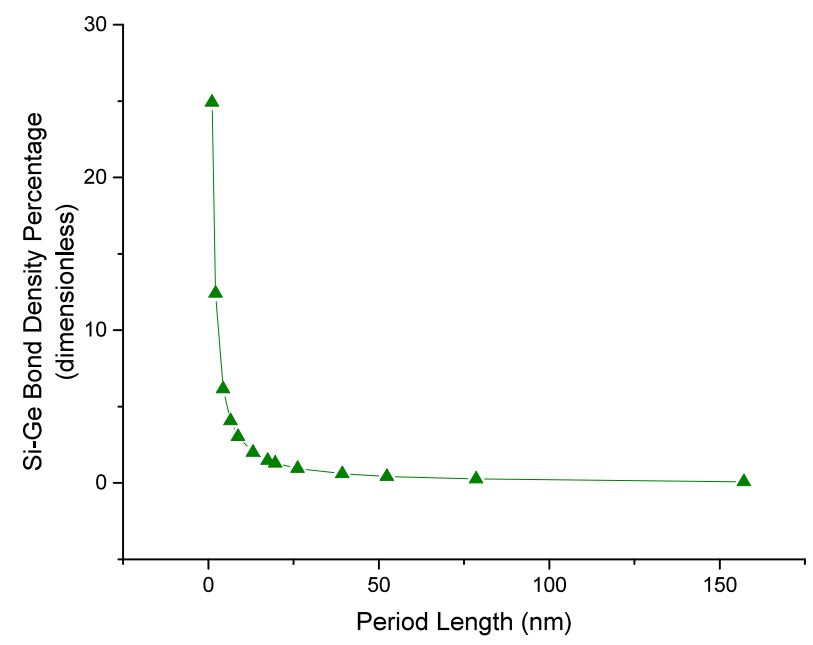

Figure 9: Density of silicon-germanium bonds vs. superlattice period length.

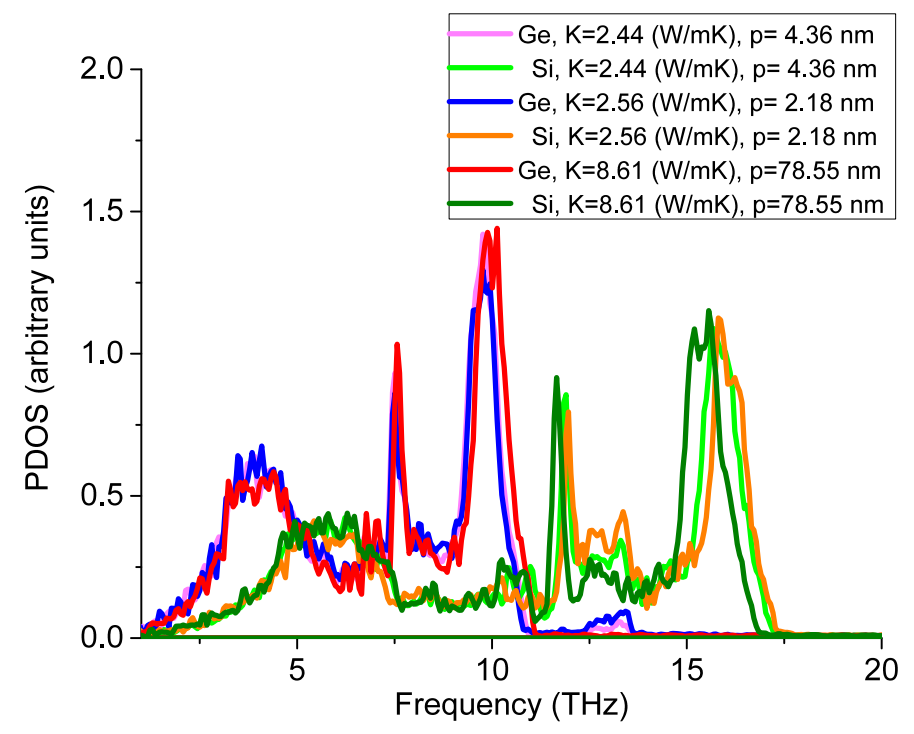

Figure 10: Phonon density of states of silicon and germanium in superlattices with various period lengths. For shorter period lengths, the number of interfaces and hence the density of interface bonds increase. Phonon properties depend on bond characteristics, thus new vibrational modes associated with interfacial bonding appear in the system. These new vibrational peaks appear between $12 \mathrm{THz}$ and $15 \mathrm{THz}$ for very short period lengths. 
modes associated with these bonds increase in amplitude. Since these new vibrational modes are present in both silicon and germanium, they can support propagating modes and hence increase thermal transport through the superlattice.

\section{Conclusions}

In this work we used molecular dynamics (MD) to simulate strain relaxation in $\mathrm{Si} / \mathrm{Ge}$ single-junction and multijunction heterostructures, focusing on its effects on thermal properties. We find that the strain relaxes within approximately two nanometers of the interface. Thus, for superlattices with a period length smaller than four nanometers the strain does not fully relax and hence the lattice structure of the superlattice is more uniform. TBR is not greatly affected by nonzero strain relaxation lengths but rather appears to depend primarily on the lattice properties within a few nanometers of the junction. A minimum in superlattice thermal conductivity versus period length is observed at a period length comparable to the strain relaxation length, implying that the increase in thermal conductivity for shorter periods may be due in part to a lack of strain relaxation (leading to a more uniform lattice constant).

We calculated the PDOS of silicon and germanium in superlattices with different period lengths, observing a new peak for short period lengths that arises due to a large density of interfacial bonds. This new peak occurs in both silicon and germanium between $12 \mathrm{THz}$ and $15 \mathrm{THz}$ and may offer an additional channel for phonon transport within the superlattice. 


\section{Acknowledgments}

This work used the Extreme Science and Engineering Discovery Environment (XSEDE), which is supported by National Science Foundation under grant number OCI-1053575.

\section{References}

[1] R. Venkatasubramanian, E. Siivola, T. Colpitts, and B. O'Quinn, Nature 413, 597 (2001)

[2] F. Capasso, A. Tredicucci, C. Gmachl, D. L. Sivco, A. L. Hutchinson, A. Y. Cho, and G. Scamarcio, IEEE Journal of Selected Topics in Quantum Electronics 5, 792-807 (1999)

[3] A. Tredicucci, C. Gmachl, F. Capasso, D. L. Sivco, A. L. Hutchinson, and A. Y. Cho, Letters to Nature 396, 350-353 (1998)

[4] C. H. Ahn, K. Senthil, H. K. Cho, and S. Y. Lee, Scientific Reports 3, $2737(2013)$

[5] S. T. Huxtable, A. R. Abramson, C.-L. Tien, A. Majumdar, C. LaBounty, X. Fan, G. Zheng, J. E. Bowers, and E. T. Croke, Applied Physics Letters 80, 1737-1739 (2002)

[6] M. Hu and D. Poulikakos, Nano Letters 12, 5487 (2012)

[7] G. Xie, Y. Guo, B. Li, L. Yang, K. Zhang, M. Tang, and G. Zhang, Phys. Chem. Chem. Phys. 15, 14647-14652 (2013) 
[8] Y. Chen, D. Li, J. R. Lukes, Z. Ni, and M. Chen, Physical Review B $\mathbf{7 2}, 174302(2005)$

[9] S. W. Bedell, K. Fogel, D. K. Sadana, and H. Chen, Applied Physics Letters 85, 5869 (2004)

[10] J. Welser, J. L. Hoyt, S. Takagi, and J. F. Gibbons, IEDM Technical Digest, 373(1994)

[11] N. Khan, and J. Li, Applied Physics Letters 89, 151916 (2006)

[12] D. J. Singh, Q. Xu, and K. P. Ong, Applied Physics Letters 104, 011910 $(2014)$

[13] H. Peelaers, and C. G. Van de Walle, Physical Review B 86, 241401(R) (2012)

[14] k. Xiong, X. Liu, Ch. Li, and J. Gu, Computational Materials Science 99, 47-56 (2015)

[15] Ch. Yang, Zh. Yu, P. Lu, Y. Liu, H. Ye, and T. Gao, Computational Materials Science 95, 420-428 (2014)

[16] X. Zhang, C. Ying, Sh. Quan, G. Shi, and Zh. Li, Computational Materials Science 58, 12-16 (2012)

[17] X. Li, K. Maute, M. L. Dunn, and R. Yang, Physical Review B 81 , $245318(2010)$

[18] W. P. Hsieh, A. S. Lyons, E. Pop, P. Keblinski, and D. G. Cahill, Physical Review B 84, 184107 (2011) 
[19] M. Shen, W. J. Evans, D. Cahill, and P. Keblinski, Physical Review B 84, $195432(2011)$

[20] E. S. Landry and A. J. H. McGaughey, Physical Review B 80, 165304 (2009)

[21] Y. Chalopin, K. Esfarjani, A. Henry, S. Volz, and G. Chen, Physical Review B 85, 195302 (2012)

[22] X. Zhou, R. Jones, C. Kimmer, J. Duda, and P. Hopkins, Physical Review B 87, 094303 (2013)

[23] A. J. H. McGaughey and M. Kaviany, Advances in Heat Transfer 39, 169 (2006)

[24] F. H. Stillinger and T. A. Weber, Physical Review B 31, 5262 (1985)

[25] B. Qiu, H. Bao, G. Zhang, Y. Wu, and X. Ruan, Computational Materials Science 53, 278-285 (2012)

[26] T. Schneider and E. Stoll, Physical Review B 17, 1302 (1978)

[27] M. F. A. Alias, N. N. Rammo, and M. N. Makadsi, Renewable Energy 24, $347(2001)$

[28] A. A. Selezeneva, A. Y. Aleinikova, P. V. Ermakova, N. S. Ganchuka, S. N. Ganchuka, and R. E. Jones, Physics of the Solid State 54, 462 (2012)

[29] S. Plimpton, Journal of Computational Physics 117, 1 (1995) 
[30] T. F. Wietler, E. Bugiel, and K. R. Hofmann, Materials Science in Semiconductor Processing 9, 659 (2006)

[31] S-P. Ju, M-H. Weng, W-J. Lee, and J-S. Lin, Computational Materials Science 42, 595-599 (2008)

[32] T. L. See, R. X. Feng, Ch. Y. Lee, and Z. H. Stachurski, Computational Materials Science 59, 152-157 (2012) 


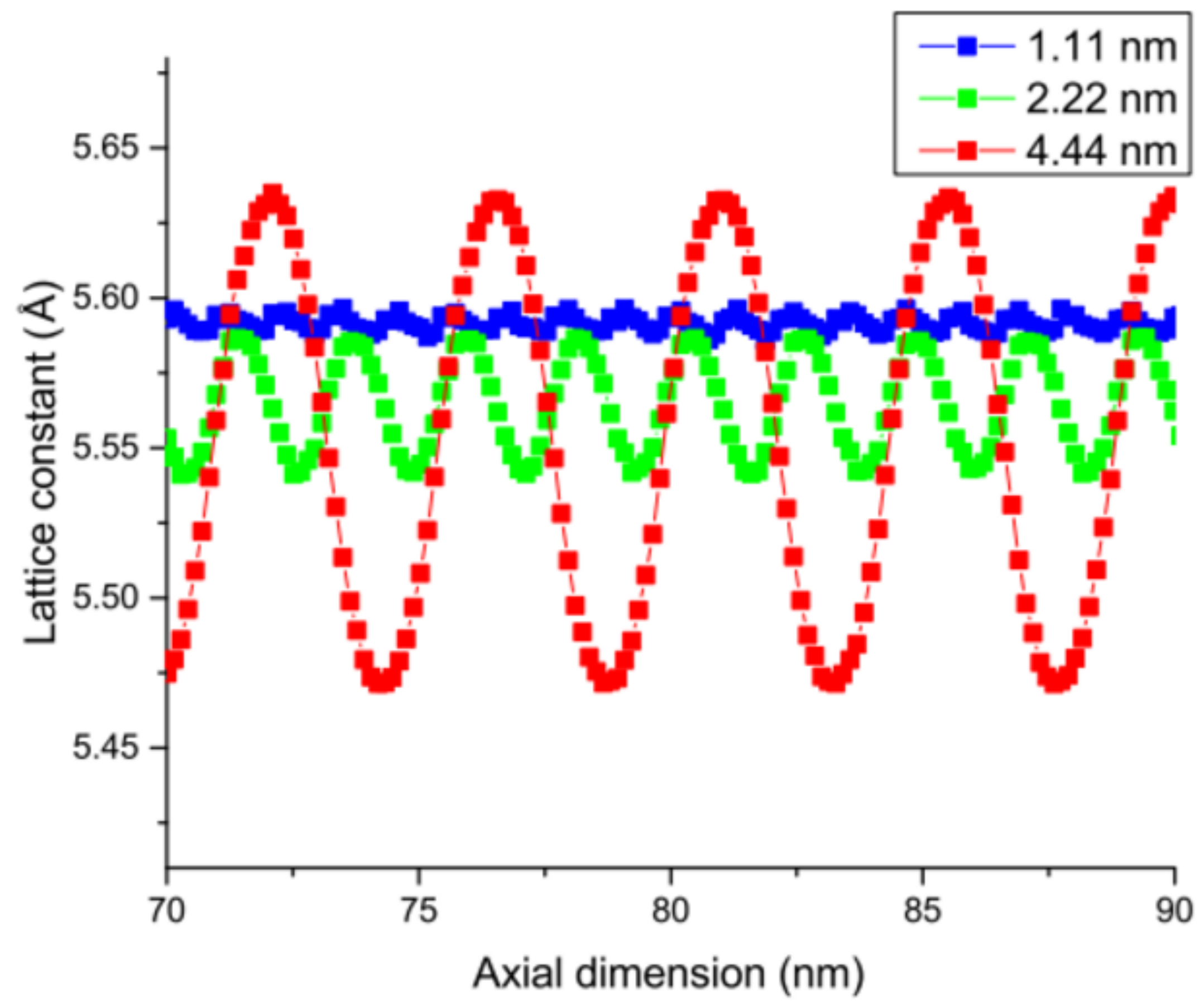

